\title{
Stiction of surface micromachined structures after rinsing and drying: model and investigation of adhesion mechanisms
}

\author{
Rob Legtenberg, Harrie A.C. Tilmans, Job Elders and Miko Elwenspoek \\ MESA Research Institute, University of Twente, PO Bor 217, 7500 AE Enschede (Netherlands)
}

\begin{abstract}
The mechanisms causing stiction of polysilicon structures fabricated by surface micromachining techniques have been investigated. It is found that during drying from rinse liquids attractive dynamic capillary forces are responsible for bringing micromechanical structures into contact with the underlying substrate. Measured adhesion energies of sticking microbridges indicate that van der Waals forces are responsible for the stiction of hydrophobic surfaces and that hydrogen bridging is an additional adhesion mechanism for hydrophilic surfaces. Methods to reduce the stiction problem are indicated.
\end{abstract}

\section{Introduction}

A notorious problem of surface micromachined structures using the sacrificial layer etching technique is the permanent attachment of slender structures to the underlying substrate after drying. This phenomenon is known as sticking or stiction [1-6]. It is assumed that during drying attractive capillary forces bring micromechanical structures into contact with the substrate. After complete drying the structures remain stuck to the substrate. It has been suggested that etch residues $[1,2]$, electrostatic forces [3] and condensation of water between the structures $[4,5]$ may be responsible for sticking. Understanding of the sticking phenomena is important for both static (beams) and dynamic (rotors) applications of surface micromachined structures with respect to technological realization and tribological aspects.

In this paper a model is presented which describes the pull-in behaviour of beams during drying from liquids, characterized by a pull-in length defined as the maximum length beyond which beams are forced into physical contact with the underlying substrate. Surface micromachined polysilicon microbridges have been fabricated and the pull-in length has been measured. After drying, beams with a length greater than the so called detachment length remain stuck to the substrate. $\Lambda$ relation between the detachment length of microbridges and the corresponding adhesion energy is given. The resulting bond strength has been determined from measured detachment lengths and is correlated to possible adhesion mechanisms.
Theory

\section{Pull-in length}

During drying, the surface tension of the diminishing liquid induces an attractive capillary force which is inversely proportional to the gap spacing between the beam and the substrate, see Fig. 1 . The restoring force consists of the bending and stretching stiffness of the beam. An increase in the deflection of the beam results in a decrease of the gap spacing and thus an increase of the capillary force. If the restoring force is not able to counterbalance the capillary force, the deflection does not reach an equilibrium position and increases until physical contact with the substratc is made. This behaviour is similar to the electrostatic attraction between two conducting surfaces as a result of an applied d.c. voltage as described in ref. 7 and can be modelled in a similar way. In the following analysis, the process is assumed to be quasi-static.

The differential equation for the static deflection $w(x)$ of a prismatic wide beam subjected to an axial (tensile) load $N$ and a transverse capillary pressure $q$ is given by:

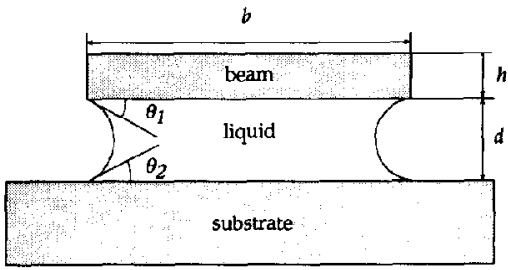

Fig. 1. Cross section of the beam during drying. 
$\hat{E} I \frac{\partial^{4} w(x)}{\partial x^{4}}-N \frac{\partial^{2} w(x)}{\partial x^{2}}=q=\frac{\gamma(\cos \theta 1+\cos \theta 2) b}{d-w(x)}$

where $\hat{E}=E /\left(1-\nu^{2}\right)$ is the plate modulus and $E$ and $\nu$ are Young's modulus and Poisson's ratio of the beam material, respectively, $I=b h^{3} / 12$ is the second moment of inertia, $\gamma$ is the surface tension of the liquid, $\theta 1$ is the contact angle between the liquid and the beam, $\theta 2$ is the contact angle between the liquid and the substrate, $d$ is the zero displacement gap spacing, $b$ the beam width and $h$ is the beam thickness.

An approximate solution can be obtained using the Rayleigh-Ritz method. A deflection profile is assumed which satisfies the geometric boundary conditions (zero displacement and rotation at the supports). The total potential energy $\Pi$ can be expressed as:

$\Pi=U_{\text {bending }}+U_{\text {stretehing }}+U_{\text {surface tension }}$

where $U_{\text {bending, }} U_{\text {stretching }}$ are the strain energy terms due to bending and stretching, respectively, and $U_{\text {surface tension }}$ is the potential energy due to the surface tension or capillary force of the fluid, given by:

$U_{\text {bending }}=\frac{1}{2} \int_{0}^{l} \hat{E} I\left(\frac{d^{2} w(x)}{d x^{2}}\right)^{2} d x$

$U_{\text {stretching }}=\frac{1}{2} \int_{0}^{l} N\left(\frac{d w(x)}{d x}\right)^{2} d x$

$U_{\text {surface tension }}=-\int_{0}^{l} \int_{0}^{w(x)} \frac{\gamma(\cos \theta 1+\cos \theta 2) b}{(d-w)} d w d x$

where $l$ is the beam length.

Trigonometric functions are very convenient for solving problems of clamped-clamped beams. An appropriate deflection profile is:

$w(x)=a\left(1-\cos \frac{2 \pi x}{l}\right)$

where $a$ is a coefficient that has to be determined. For a system at equilibrium, the first derivative of the total energy with respect to $a$ is equal to zero. For a stable equilibrium the total potential energy must be at a (local) minimum, implying that the second derivative with respect to $a$ has to be positive. The transition to an unstable system occurs when the second derivative equals zero. Solving these equations simultaneously yields the following expression for the pull-in length $L_{\mathrm{PI}}$ and the corresponding value for $a$ :

$L_{\mathrm{PI}}=\left\{\pi^{2} C d^{2}\left[N+\left(\frac{8 \hat{E} I}{C d^{2}}+N^{2}\right)^{1 / 2}\right]\right\}^{1 / 2}$

where
$C=\frac{0.1549}{r(\cos \theta 1+\cos \theta 2) b}$

and

$a=0.2950 d$

The effect of the geometry and the residual strain $\epsilon=N / E b h$ on the pull-in length is shown in Fig. 2. For the condition that $8 \hat{E} I / C d^{2} \gg N^{2}$, the influence of the residual strain on the pull-in length can be neglected and expression (7) becomes:

$L_{\mathrm{PI}}=1.059\left[\frac{8 \hat{E} d^{2} h^{3}}{\gamma(\cos \theta 1+\cos \theta 2)}\right]^{1 / 4}$

Note that the bracketed expression on the right-hand side of eqn. (8) gives the pull-in length in case the problem is simplified and the beam is modelled as a spring-mass system. The spring stiffness $k$ for this special case is given by $k=384 E I / l^{3}$ and the active area is equal to $b l$.

\section{Detachment length}

If the beam length exceeds the pull-in length physical contact with the substrate will be made during drying from the liquid. Physical contact, between the released microstructures and the underlying substrate, in turn induces an adhesive bond. If the strength of the adhesive bond exceeds the mechanical 'pull-off force', the structures remain permanently stuck to the substrate. The bonding strength of sticking beams can be determined from their detachment length as presented by Mastrangelo and $\mathrm{Hsu}$ [5] for cantilever beams. It is evident that the detachment length is always equal to or larger than the pull-in length, since sticking only occurs after physical contact has been made.

Using an array of beams with increasing length the detachment length is equal to the length where a

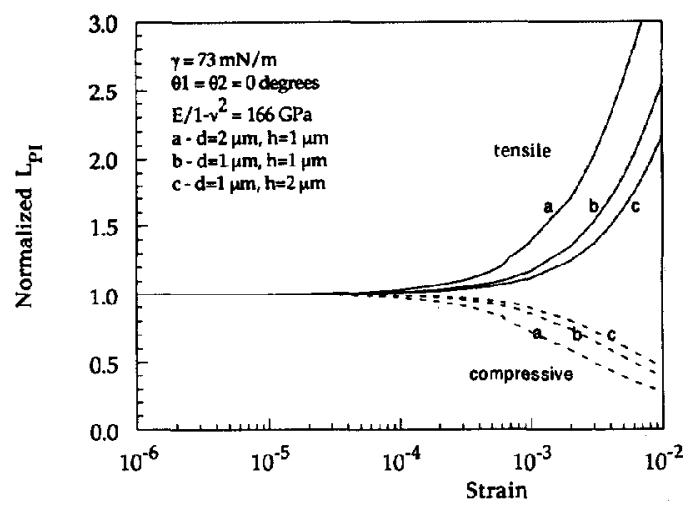

Fig. 2. The influence of the mechanical strain on the pull-in length $L_{\mathrm{PI}}$ for different beam thicknesses and gap spacing normalized on the pull-in length at zero strain. 
transition occurs from sticking beams to free standing beams. The relation between the detachment length and the surface energy is derived as follows. The stored elastic energy in doubly clamped beams again consists of a bending and stretching term, sec eqns. (3) and (4). The energy of adhesion is stored in the segment of the beam that is in physical contact with the substrate. At equilibrium the total energy of the system is minimized with respect to $s$ (see Fig. 3) and an expression for the bond strength can be found. In this case an appropriate deflection profile of the beam is:

$w(x)=\frac{1}{2} d\left(1-\cos \frac{\pi x}{s}\right)$

where $s$ is the region near the support of the beam that is not sticking. We find for the total energy of this system:

$\Pi=\frac{\pi^{4} \hat{E} I d^{2}}{8 s^{3}}=\frac{\pi^{2} N d^{2}}{8 s}-\gamma_{s}(l-2 s) b$

Equilibrium is found by setting $d U_{\text {total }} / d s=0$, yielding the value for $s$ at which the energy is minimal. The energy has a single equilibrium point if $s<l / 2$ and no equilibrium point if $s>l / 2$, i.e. the beam is sticking to the substrate in the former case and is free standing in the latter case. At the transition from sticking to free standing beams, $s$ is equal to $l / 2$ giving the following relation between the bond strength $\gamma_{s}$ and the detachment length $L_{\text {det }}$ :

$\gamma_{\mathrm{s}}=\frac{\pi^{4} \hat{E} h^{3} d^{2}}{4 L_{\mathrm{det}}{ }^{4}}+\frac{\pi^{2} \epsilon E h d^{2}}{4 L_{\mathrm{det}}{ }^{2}}$

The influence of the residual strain, both compressive and tensile, on the detachment length is very small when $\epsilon L_{\operatorname{det}}{ }^{2} / \pi^{2} h^{2} \ll 1$. In this case the second term on the right-hand side of eqn. (11) can be neglected.

\section{Adhesion of solids}

By measuring the detachment length of samples with different combinations of gap spacing and beam thickness, the bond strength can be determined. From the measured value of the bond strength, as determined from the detachment length, information about the adhesion mechanisms that are present can be deduced.

The contents of this section are based upon the adhesion theories described in refs. 8-12. Interactions between solids which bring about adhesion can be

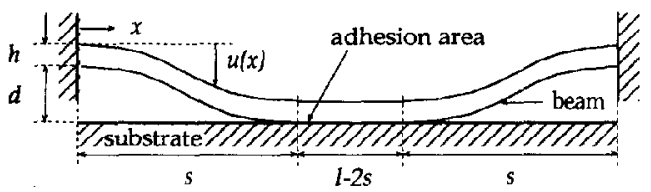

Fig. 3. Sketch of a polysilicon beam adhering to the substrate. classified as follows [8]. First there are long-range attractive interactions, including capillary forces, van der Waals forces and electrostatic forces, which establish the adhesive contact area. Secondly there are shortrange interactions caused by hydrogen bonds, chemical bonds and metallic bonds. Interfacial interactions based on diffusion and alloying are generally very slow at room temperature and will not be considered.

After HF etching, the hydrophobic silicon surface consists mainly of $\mathrm{Si}-\mathrm{H}$ bonds which are chemically stable. The silicon surface shows a logarithmic growth rate up to roughly half a monolayer equivalent of silicon oxide during exposure to water or air. This growth rate increases after $3 \mathrm{~h}$ exposure to water and after nearly one week exposure to air $[13,14]$. On further exposure the surface becomes hydrophilic. Because the surfaces are contaminated, strong ionic interactions between silicon surface atoms are screened out. Chemical reactions between surface hydroxyl groups only start to play a role at elevated temperatures [15]. For comparison, a system consisting of two solids, each one having the form of an infinitely extended half space, will be considered. The bonding energy per unit area due to the most relevant adhesion mechanisms is discussed below. Figure 4 shows the bond strength for each mechanism as a function of the separation.

\section{van der Waals forces}

van der Waals forces result from the interaction between instantaneous dipole moment of atoms and can be described by the Lifshitz theory $[8,9]$. The expression for the van der Waals energy is given by the following equation for separations in the nonretarded regime $(\delta<20 \mathrm{~nm})$ :

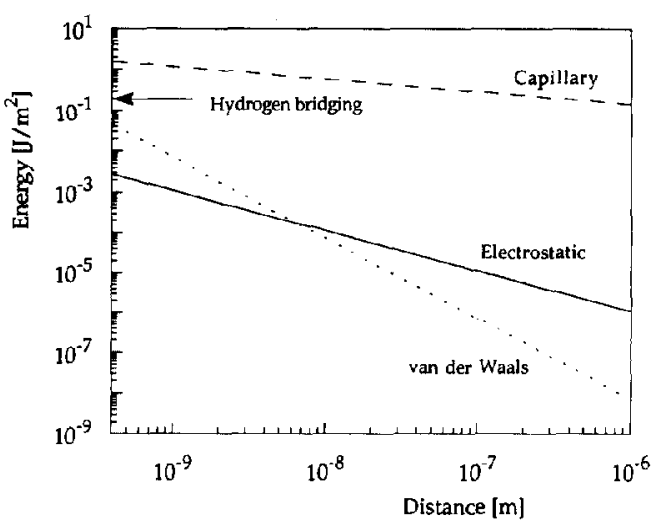

Fig. 4. Bonding energy vs. separation. The capillary energy was calculated for water using a surface tension of $73 \mathrm{mN} / \mathrm{m}$ and a contact angle of zero degrees with a reference distance $\delta_{\mathrm{r}}$ of 2 $\mu \mathrm{m}$. For the electrostatic energy a voltage difference of $0.5 \mathrm{~V}$ was used and for the van der Waals energy a Hamaker's constant of silicon in air equal to $27 \times 10^{-20} \mathrm{~J}$ was used. 


$$
E_{\mathrm{vdw}}=\frac{H}{12 \pi \delta^{2}}
$$

where $H$ is Hamaker's constant and $\delta$ is the separation distance. The surface energy due to van der Waals forces between two solid surfaces in contact can be predicted by:

$\gamma_{\mathrm{s}}=\frac{1}{2} E_{\mathrm{vdw}}=\frac{H}{24 \pi D_{0}^{2}}$

where $D_{0}$ is a cut-off distance of about $0.2 \mathrm{~nm}$ [9]. This yields a surface energy for silicon of $90 \mathrm{~mJ} / \mathrm{m}^{2}$ using a Hamaker's constant of silicon in air of $27 \times 10^{-20}$ J [18].

\section{Capillary forces}

The energy as a result of capillary forces is given by:

$E_{\text {capillary }}=\int_{\delta}^{\delta_{r}}-\frac{\gamma(\cos \theta 1+\cos \theta 2)}{\delta} d \delta$

where $\gamma$ is the surface tension of the liquid and $\theta 1$ and $\theta 2$ are the contact angles of the liquid with the two surfaces. As opposed to the other mechanisms, the reference distance $\delta_{\mathrm{r}}$ of the capillary energy cannot be chosen as infinite but it is readily shown that, under conditions of meniscus forces, the capillary energy exceeds all other adhesive components (see Fig. 4). When two hydrophilic surfaces are in or close to contact in a humid environment, water will capillary condense in the small gap between them until the meniscus radius reaches the Kelvin radius $r_{K}$ as determined by the Kelvin equation [9].

\section{Electrastatic forces}

Electrostatic forces can arise from Coulomb attraction between charged objects or from the contact potential between two surfaces caused by differences in the local energy states and electron work functions. Its energy is given by:

$E_{\mathrm{el}}=\frac{\epsilon_{0} U^{2}}{2 \delta}$

where $U$ is the potential difference between the surfaces separated by an air gap with permittivity $\epsilon_{0}$. At small separations electrostatic pressures equal van der Waals pressures only for extremely large surface charge densities $\left(>10^{13}\right.$ elementary charges per $\left.\mathrm{cm}^{2}\right)$ and large contact-potential differences $(>0.5 \mathrm{~V})$ and are unlikely ever to exceed them [8], see Fig. 4.

\section{Hydrogen bridging}

Hydrophilic silicon surfaces contain a large number of hydroxyl groups. The hydroxyl groups can form strong hydrogen bonds as the separation between both surfaces becomes small. The density of hydrogen-bonding sites is given by the number of silanol groups and is found to be $5.0 \pm 0.5$ per $\mathrm{nm}^{2}$ for fully hydrated silica surfaces [16]. The strength of most hydrogen bonds lies between 10 and $40 \mathrm{~kJ} / \mathrm{mol}$ [9], thus yielding adhesion energies between 0.1 and $0.3 \mathrm{~J} / \mathrm{m}^{2}$ for these surfaces.

Besides the above mentioned bonding mechanisms Alley et al. [2] suggested that polysilicon structures stick together due to a silicon oxide residue, left behind after a chemical reaction of the silicon surface with water. In this case an intermediate material is introduced between the silicon substrate surface and the polysilicon beam surface. The adhesive strength of solid bridging is high [17] but difficult to estimate and is dependent on the type and amount of residue material.

In addition, all attractive forces except the meniscus forces decrease rapidly with increasing distance.

In practice, the surfaces exhibit a surface roughness which strongly influences the real contact area and nominal separation [18]. The surface roughness has a significant effect on the adhesion of elastic solids [19] and small increases of the surface roughness are sufficient to reduce the adhesion to very small values.

\section{Experiments}

\section{Fabrication}

Test structures consisting of an array of doubly clamped undoped polysilicon beams were fabricated. First, a PECVD silicon oxide layer was grown on an n-type (100) silicon wafer $(5-10 \Omega \mathrm{cm})$ from a $2 \% \mathrm{SiH}_{4}$ in $\mathrm{N}_{2}$ gas mixture and $\mathrm{N}_{2} \mathrm{O}$ gas at a pressure of 650 mTorr and a temperature of $300{ }^{\circ} \mathrm{C}$. This layer was patterned using BHF to form the beam anchors. Next, an undoped polysilicon layer was grown by LPCVD from $\mathrm{SiH}_{4}$ at a pressure of $250 \mathrm{mT}$ Torr and a temperature of $590^{\circ} \mathrm{C}$. The wafers were then annealed at $1100{ }^{\circ} \mathrm{C}$ in $\mathrm{N}_{2}$ for $1 \mathrm{~h}$ to obtain a low residual stress (typically in the order of $10 \mathrm{ppm}$ ). The polysilicon layer was patterned by RIE using an $\mathrm{SF}_{6}$ plasma to form the beams with lengths ranging from 10 to $200 \mu \mathrm{m}$ in steps of $1 \mu \mathrm{m}$ and widths of 20 and $50 \mu \mathrm{m}$. The polysilicon and the silicon oxide thickness ranged from 0.5 to 2 $\mu \mathrm{m}$ in steps of $0.5 \mu \mathrm{m}$ resulting in a $4 \times 4$ matrix of the gap spacing $d$ and the beam thickness. $h$. Samples were cleaned prior to sacrificial layer etching. Sacrificial layer etching was done in a $50 \%$ HF solution for 25 min. After this, different treatments were used. Special care was taken to keep the wafers wet and as clean as possible. Dilution rinsing for $15 \mathrm{~min}$ in DI water and drying yields hydrophobic wafers dried from $\mathrm{H}_{2} \mathrm{O}$. Adding isopropylalcohol (IPA) to the water keeps the 
wafers wet after removal from the solution. Next they were rinsed in IPA for $15 \mathrm{~min}$ and withdrawn from the liquid. This procedure gives hydrophobic wafers dried from IPA. A set of hydrophilic wafers was also produced by adding nitric acid after DI dilution rinsing. At a concentration of $70 \%$ nitric acid the solution was heated to $100{ }^{\circ} \mathrm{C}$ for $1 \mathrm{~h}$ to render the silicon surface hydrophilic. After dilution rinsing for $15 \mathrm{~min}$ in DI water a set of hydrophilic wafers dried from $\mathrm{H}_{2} \mathrm{O}$ was obtained. The same procedure followed by an IPA rinse as described above gives hydrophilic wafers dried from IPA.

\section{Measurements}

\section{Pull-in length}

To verify the model for the pull-in length, hydrophobic and hydrophilic wafers dried from IPA were used. Wet samples were placed under a microscope connected to a video camera system. The drying process was recorded using interference contrast and pull-in was visible during slow replay. The shortest beam showing pull-in due to the surface tension represents the pull-in length. The results are shown in Fig. 5.

The contact angle of a droplet of IPA on a hydrophobic or hydrophilic silicon surface was measured to be smaller than $5^{\circ}$. The measured contact angles of water on hydrophobic silicon and water on hydrophilic silicon were $70 \pm 5$ and $5 \pm 5^{\circ}$, respectively.

\section{Detachment length}

After drying, not all the beams that pulled-in during drying deflect upward again but some remain permenently attached to the substrate. The transition from sticking to free standing beams after drying, the detachment length, was also determined by interference

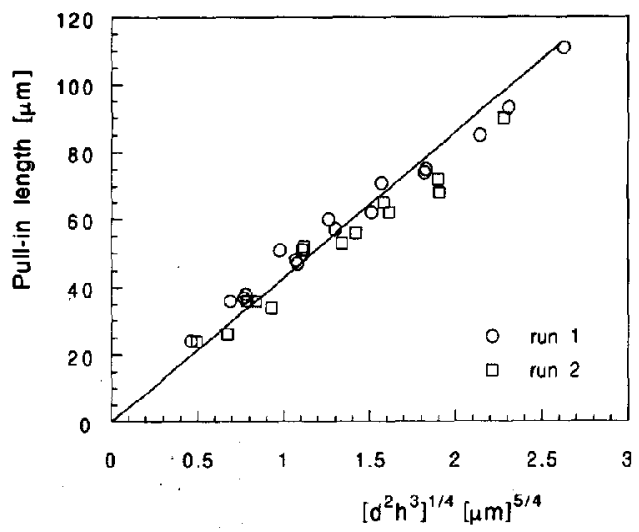

Fig. 5. Measured pull-in length as a function of $\left[d^{2} h^{3}\right]^{1 / 4}$ for polysilicon beams dried from IPA. Measurements were performed on two separate fabrication runs, each resulting in a $4 \times 4$ matrix of polysilicon beams with a gap spacing $d$ and a beam thickness $h$. contrast microscopy (see Fig. 6). For the detachment length experiments samples were dried from two commonly used rinsing liquids, namely $\mathrm{H}_{2} \mathrm{O}$ and IPA, to see whether or not the rinse liquid has an effect on the detachment length. Drying from these solutions was done for hydrophobic and hydrophilic samples. The results of these measurements are shown in Fig. 7.

The residual strain of the polysilicon layer was measured by ring and buckled beam structures and was typically found to be in the order of $10 \mathrm{ppm}$. For the dimensions of the samples in this paper and the measured residual strain, the conditions to neglect the influence of the residual strain are generally satisfied (see previous section).

The detachment length showed scattering especially for the hydrophobic samples dried from water. Often long beams are free standing while shorter beams are sticking. Disturbances because of local surface asperities, dust particles, etc. strongly reduce adhesion re-

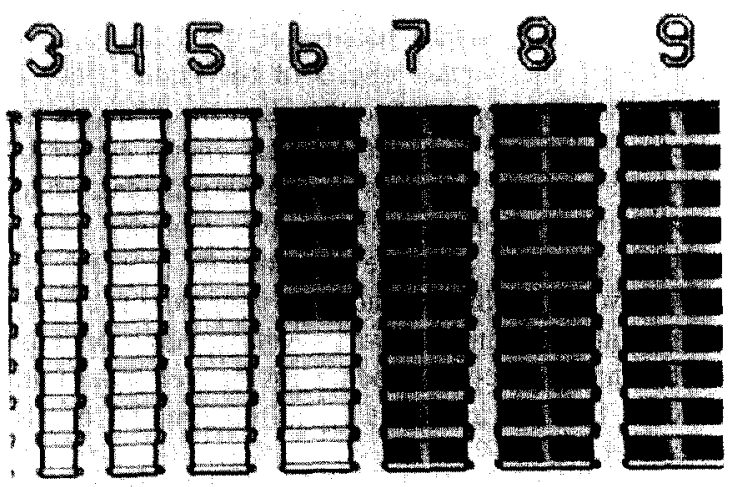

Fig. 6. Photograph showing the transition from free standing to sticking beams which is clearly visible by interference patterns.

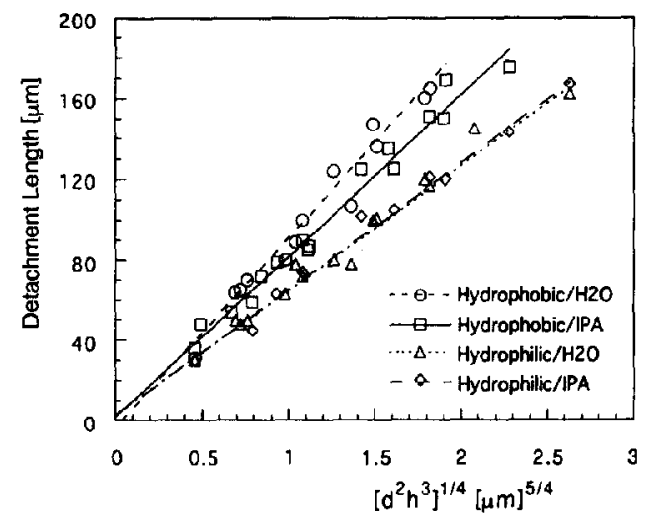

Fig. 7. Detachment length as a function of $\left[d^{2} h^{3}\right]^{1 / 4}$ for hydrophobic and hydrophilic samples dried from water $\left(\mathrm{H}_{2} \mathrm{O}\right)$ and isopropanol (IPA). 
sulting in errors that lead to an increase of the detachment length. In our experiments, the detachment length was determined from the shortest sticking beams (i.e. giving the highest bonding strength).

From the detachment length the surface energy can be calculated by eqn. (11). The slope of the curves in Fig. 7 is equal to $\left[\pi^{4} E / 4 \gamma_{\mathrm{s}}\right]^{1.4}$. The corresponding surface energies for hydrophobic and hydrophilic samples are listed in Table 1 . The errors in the surface energies are worst case values and are mainly determined by the error in the slopes of the curve fits.

Some samples were inspected by SEM to see if etch residues were left behind after the etching and rinsing procedures, but only smooth and clean surfaces were observed. Figure 8 shows an SEM photograph of the disappearing gap spacing between a sticking beam and the substrate.

Because the roughness is important with respect to the adhesion properties, as discussed before, the surface roughness was measured by atomic force microscopy. The root mean square values of the surface roughness of the bottom side of the microbridges varied from about 1 to $3 \mathrm{~nm}$, depending on the thickness of the silicon oxide and polysilicon layer. The surface roughness of the silicon substrate after sacrificial layer etching

TABLE 1. Surface energy of the different samples calculated from formula (11). A value of $166 \mathrm{GPa}$ was used for the plate modulus $E$. Residual strain is in the order of $10 \mathrm{ppm}$ and can be neglected in our experiments

\begin{tabular}{lll}
\hline Liquid & \multicolumn{2}{l}{ Surface energy $\left(\mathrm{J} / \mathrm{m}^{2}\right)$} \\
\cline { 2 - 3 } & Hydrophobic & Hydrophilic \\
\hline $\mathrm{H}_{2} \mathrm{O}$ & $0.05 \pm 0.05$ & $0.26 \pm 0.1$ \\
IPA & $0.10 \pm 0.05$ & $0.24 \pm 0.1$ \\
\hline
\end{tabular}

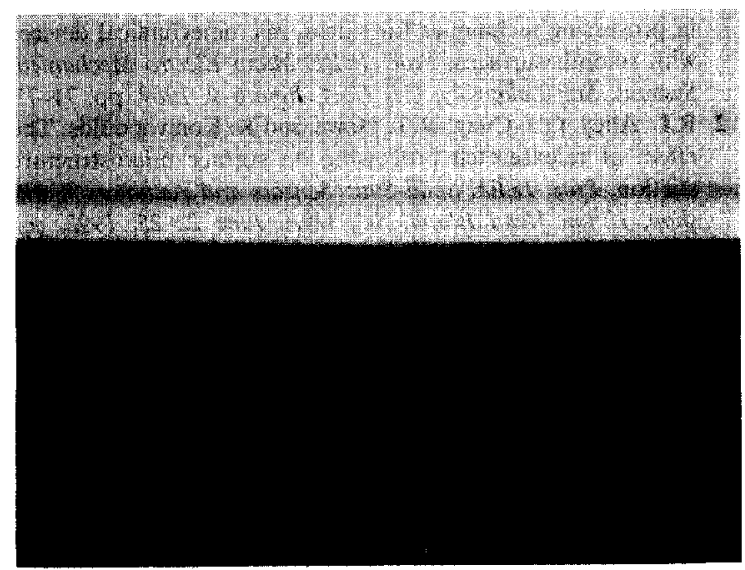

Fig. 8. SEM photograph of the disappearing gap spacing between a sticking beam and the substrate. was $0.2 \mathrm{~nm}$. These values are very small and imply that the measured surface energies are only weakly influenced by the surface roughness of the samples.

\section{Discussion}

From Fig. 5 it can be seen that the measured pull-in length shows a linear fit with the parameter $\left[d^{2} h^{3}\right]^{1 / 4}$ as predicted by the theory. However, the slope of the curve is $41 \mu \mathrm{m}^{-1 / 4}$, which is lower than the theoretical value of $79 \mu^{-1 / 4}$, calculated from eqn. (8) by using a plate modulus $\hat{E}$ of $166 \mathrm{GPa}$, a surface tension $\gamma$ for IPA of $21.7 \mathrm{mN} / \mathrm{m}$ [20] and zero degree contact angles $\theta 1$ and $\theta 2$. The influence of the stepup boundary of the fabricated microbeams or other assumptions for the deflection profile have been considered and only lead to small variations in the pullin length and are thus unlikely to cause the discrepancy. The difference might be due to the non-equilibrium situation that exist during drying. The value used for the surface tension is only valid for IPA at thermodynamical equilibrium. During drying however, the liquid is not in equilibrium. Dynamic effects such as spreading may lead to in an increase of the capillary forces. In the case of spreading [21], the difference can be enormous, and is about $60 \mathrm{mM} / \mathrm{m}$ for organic liquids on oxides and $300 \mathrm{mN} / \mathrm{m}$ for water on metallic oxides. In our situation the increase would be $270 \mathrm{mN} /$ $\mathrm{m}$ as determined from the pull-in measurements.

The measured surface energies of sticking beams (Table 1) can be compared with the theory to determine which adhesion mechanisms are present. After the rinsing and drying procedures, as described above, no residues have been observed by microscopic or SEM inspection indicating that adhesion by solid bridging is not present. From Table 1 we see that the rinsing liquids which have been used hardly effect the adhesion energy and that it is mainly determined by the surface properties (i.e. hydrophobic or hydrophilic) of the materials which have been used. Although meniscus forces play an important role in the initiation of the physical contact between the beam and the substrate, the measured bond energies are too small to be caused by meniscus forces of a thin liquid film, remaining after drying or initiated by microcapillary effects. Also chemical bonding is precluded for the same reason. As already discussed before, electrostatic forces are unlikely to exceed van der Waals forces at small separations and are disregarded as a possible cause for stiction. This leaves van der Waals forces and hydrogen bonding as the possible causes for stiction.

The measured surface energies indicate that van der Waals forces are responsible for the hydrophobic bonded surfaces and hydrogen bridging is an additional bond 
mechanism for the hydrophilic bonded surfaces. The values for the surface energy show a good correlation with hydrophobic and hydrophilic wafer bonding experiments [15]. In this case energies are found of 0.04 to $0.1 \mathrm{~J} / \mathrm{m}^{2}$ for hydrophobic wafers and 0.14 to $0.2 \mathrm{~J} /$ $\mathrm{m}^{2}$ for hydrophilic wafers bonded at room temperature.

In summary, stiction is accomplished in a two-step process. First a temporary physical contact between surface micromachined structures and the substrate is induced by dynamic capillary forces during drying of the rinse liquid. After this, permanent attachment is caused by van der Waals forces and hydrogen bridges which are induced by the small separation distance during this contact.

The maximum free standing length, the detachment length, is a function of the adhesion energy of the beam to the substrate surface and the mechanical stiffness of the structures. The maximum free standing length can easily be increased by increasing the beam thickness and the sacrificial layer thickness and using larger tensile residual strains.

Stiction can be prevented or reduced in several ways which can roughly be divided into two groups. First there are methods based upon the prevention of physical contact between the structures and the substrate during fabrication. This is done by avoiding the attractive meniscus forces that bring the structures in contact with the substrate during the drying process, e.g. by freeze drying $[1,22,23]$, critical point drying [24] or dry etching techniques $[25,26]$. Also temporary increasing the mechanical stiffness of the structures by using breakaway supports [27] has been used. These methods have the disadvantage that stiction can still occur if the structures are afterwards brought into contact with the substrate by external forces like large accelerations or static charges [23]. Second there are methods based upon the reduction of the adhesional forces. This can be done by minimizing the surface energy, for instance by using hydrophobic surfaces or by surface treatments [2], and by reduction of the real contact area by using stand-off bumps $[6,28]$ or by increasing the surface roughness [29]. These methods result in a permanent reduction of the adhesional forces.

\section{Conclusions}

It has been shown that during the drying process, attractive capillary forces are responsible for bringing micromechanical structures into contact with the substrate. A model for the surface tension forces during drying has been developed and applied to doubly clamped beams. For these beams a pull-in length exists beyond which the beams are forced into contact with the underlying substrate. Measurements of the pull-in length are in qualitative agreement with the theory. The measured values of the pull-in length are somewhat lower than predicted by theory. This is probably due to dynamical effects during the drying process.

After complete drying, structures remain stuck to the substrate. The measured adhesion energy of the resultant bond has been determined using the detachment length of the beams after complete drying. The resulting bonding strengths were independent of the used rinsing liquids, water and isopropanol. The adhesion energy ranged from 0.05 to $0.10 \mathrm{~J} / \mathrm{m}^{2}$ for hydrophobic silicon surfaces and from 0.24 to $0.26 \mathrm{~J} / \mathrm{m}^{2}$ for hydrophilic silicon surfaces. These energies indicate that van der Waals forces are responsible for the stiction of hydrophobic surfaces and hydrogen bridging is the dominant bond mechanism in the case of stiction of hydrophilic surfaces. These results are in agreement with dry wafer bonding experiments. Several methods to prevent or reduce stiction have been discussed.

\section{Acknowledgements}

The authors thank Erwin Berenschot and Meint de Boer for sample preparation, Bert Otter for his help with the AFM measurements and the SEM photographs, and Geert Langereis for doing the pull-in length measurements. This work is part of the program of the Dutch Foundation for Fundamental Research on Matter (FOM) and is sponsored by the Dutch Technology Foundation (STW).

\section{References}

1 H. Guckel, J.J. Sniegowski and T.R. Christenson, Advances in processing techniques for silicon micromechanical devices with smooth surfaces, Proc. IEEE Micno Electro Mechanical Systems, Salt Lake City, UT, USA, June 6-9, 1989, pp. 71-75.

2 R.L. Alley, G.J. Cuan, R.T. Howe and K. Komvopoulos, The effect of release-etch processing on surface microstructure stiction, Proc. IEEE Solid-State Sensors and Actuators Workshop, Hilton Head Island, SC, USA, June 22-25, 1992, pp. 202-207.

3 C. Linder, E. Zimmerman and N.F. de Rooy, Capacitive polysilicon resonator with MOS detection circuit, Sensor and Actuators A, 25-27 (1991) 591-595.

4 P.R. Scheeper, J.A. Voorthuyzen, W. Olthuis and P. Bergveld, Investigation of attractive forces between PECVD silicon nitride microstructures and an oxidized silicon substrate, Sensors and Actuators A, 30 (1992) 231-239.

5 C.H. Mastrangelo and C.H. Hsu, A simple experimental technique for the measurement of the work of adbesion of microstructures, Proc. IEEE Solid-State Sensors and Actuators Workshop, Hitton Head Island, SC, USA, June 22-25, 1992, pp. 208-212. 
6 W.C. Tang, T.C.H. Nguyen and R.T. Howe, Laterally driven polysilicon resonant microstructures, Proc. IEEE Micro Electro Mechanical Systems, Salt Lake City, UT, USA, Feb. 20-22, 1989, pp. 53-59.

7 H.A.C. Tilmans, Micro-mechanical sensors using encapsulated built-in resonant strain gauges, $P h . D$. Thesis, University of Twente, Jan. 1993, Ch. 4.

8 H. Krupp, Adv. Colloid Interface Sci, 1 (1967) 111-239.

9 J. Isrealachvilli, Intermolecular and Surface Forces, Academic Press, San Diego, 2nd edn., 1991.

10 J. Isrealachvilli, P. McGuiggan and R. Horn, Basic physics of interaction between surfaces in dry, humid and aqueous environments, Proc. Electrochem. Soc. First Int. Symp. Semiconductor Wafer Bonding, Phoenix, AZ, USA, Oct. 13-18, 1991, pp. 33-47.

11 A.D. Zimon, Adhesion of Dust and Powders, Consultants Bureau, New York, 2nd edn., 1979.

12 R.A. Bowling, An analysis of particle adhesion on semiconductor surfaces, J. Electrochem. Soc., 132 (1985) 2208-2214.

13 D. Gräf, M. Grundner and R. Schultz, The reaction of water with bydrofluoric acid treated silicon (111) and (100) surfaces, J. Vac Sci. Technol., A7 (1989) 808-813.

14 M. Grundner, D. Gräf, P.O. Hahn and A. Schnegg, Wet chemical treatment of Si surfaces: chemical composition and morphology, Solid State Technol, (Feb.) (1991) 69-75.

15 Y. Bäcklund, K. Herrmansson and L. Smith, Bond strength measurements related to silicon surface hydrophilicity, Proc. Electrochem. Soc. Fint Int. Symp. Semiconductor Wafer Bonding, Phoenix, AZ, USA, Oct. 13-18, 1991, pp. 82-87.

16 R.K. Iler, The Chemistry of Silica, Wiley, New York, 1979, P. 177

17 A. Torri, M. Sasaki, K. Hane and S. Oluma, Adbesive force of the microstructures measured by the atomic force microscope, Proc. IEEE Micro Electro Mechanical Systems, Fort Lauderale, FL, USA, Feb. 7-10, 1993, pp. 111-116.

18 J.A. Greenwood and J.B.P. Williamson, Contact of nominally fiat surfaces, Proc. R. Soc. London, Ser. A, 295 (1966) 300-319.

19 X.N.G. Fuller and D. Tabor, The effect of surface roughness on the adhesion of elastic solids, Proc. $R$. Soc. London, Ser. A, 345 (1975) 327-342.

20 Handbook of Chemistry and Physics, CRC Press, Boca Raton, FL, 66th edn., 1985-1986.

21 P.G. de Gennes, Wetting: statics and dynamics, Rev. Mod. Phys., 57 (1985) 827-863.

22 N. Takeshima, K.J. Gabriel, M. Ozaki, J. Takahasji, H. Horiguchi and $\mathbf{H}$. Fujita, Electrostatic parallelogram actuators, Proc. 6th Int. Conf. Solid-State Sensars and Acheators (Transducers '91), San Francisco, CA, USA, June 24-28, 1991, pp. 63-66.

23 R. Legtenberg and H.A.C. Tilmans, Electrostatically driven vacuum encapsulated polysilicon resonators: Part I. Design and fabrication, Sensors and Actuators, submitted for publication.

24 G.T. Mulhern, D.S. Soane and R.T. Howe, Supercritical carbon dioxide drying of microstructures, Proc. 7th Int. Conf. Solid-State Sensors and Actuators (Transducers 93), Yokohama, Japan, June 7-10, 1993, pp. 296-299.

25 M. Orpana and A.O. Korhonen, Control of residual stress of polysilicon thin films by heavy doping in surface micromachining, Proc. 6th Int. Conf. Solid-State Sensors and Actuators (Transducers '91), San Francisco, CA, USA, June 24-28, 1991, pp. 957-960.

26 D. Kobayashi, C.J. Kim and H. Fujita, Photoresist-assisted release of movable microstructures, Proc. 7th Int. Conf. SolidState Sensors and Actuators (Transducers '93), Yokohama, Japan, June 7-10, 1993, Abstr. late news papers, pp. 14-15.
27 R.A. Brennen, M.G. Lim, A.P. Pisano and A.T. Chou, Large displacement linear actuator, Proc. IEEE Solid-State Sensors and Actuators Workshop, Hilton Head Island, SC, USA, June 4-7, 1990, pp. 135-139.

28 F.S.A. Sandejas, R.B. Apte, W.C. Banyai and D.M. Bloom, Surface microfabrication of deformable grating light valves for high resolution displays, Proc. 7th Int. Conf. Solid-State Sensors and Actuators (Transducers '93), Yokohama, Japan, June 7-10, 1993, Abstr. late news papers, pp. 6-7.

29 R.L. Alley, P. Mai, K. Komvopoulos and R.T. Howe, Surface roughness modification of interfacial contact in polysilicon microstructures, Proc. 7th Int. Conf. Solid-State Sensors and Actuators (Transducers '93), Yokohama, Japan, June 7-10, 1993, pp. 288-291.

\section{Biographies}

Rob Legtenberg was born on June 21, 1964 in Hengelo, The Netherlands. He received the B.Sc. degree in applied physics, from the Hogere Technische School Enschede, Enschede, The Netherlands, in June 1985. After fulfilling his military service, he joined the Micro Mechanical Research group at the University of Twente as a process engineer in September 1986. Since April 1992 he has been preparing for his Ph.D. dealing with the development of actuators based on surface micromachining technology.

Harrie Tilmans was born on November 14, 1957 in Elsloo, The Netherlands. He received his M.Sc. degree in electrical engineering from the University of Twente, Enschede, The Netherlands, in May 1984. In June 1984 he became a temporary researcher at the University of Twente where he worked on the development of a resonating force sensor. In April 1985 he joined the faculty of Electrical and Computer Engineering of Boston University, Boston, MA, USA as a visiting instructor. In August 1986 he became a research assistant at the Wisconsin Center for Applied Microelectronics at the University of Wisconsin, Madison, WI, USA, where he worked on the development of a CMOS process and on the development of polysilicon micromechanical resonators. In August 1988 he returned to the Netherlands where he joined the MESA Research Institute of the University of Twente as a research associate to work on 'micromechanical sensors using encapsulated built-in resonant strain gauges'. He received his Ph.D. degree on this topic in January 1993. From August 1989 till January 1990 he was on leave from the university to join the Sensors group of the Controls Research Department of Johnson Controls Inc., Milwaukee, WI, USA to work on a low-range differential pressure sensor. He is currently employed by F.O.M. (Foundation for Fundamental Research on Matter, The Netherlands) on a postdoctorate position within the micromechanics group of the MESA Research 
Institute to work on the characterization of mechanical properties of thin films.

Job Elders was born on June 1, 1964 in Amsterdam, The Netherlands. He graduated in 1987 at the University of Amsterdam in chemistry on intra- and intermolecular energy transfer in supersonic molecular beams. He obtained his Ph.D. at the same university in the field of laser-induced chemical vapour deposition. Since 1992 he has been working at the MESA Research institute. His current research interests are in the field of materials analysis of thin films used for miniaturized devices.

Miko Elwenspoek graduated in 1977 from the Free University of Berlin in physics on Mößbauer scattering from highly viscous liquids. From 1977 to 1979 he worked in the group of Professor W. Helfrich on lipid membranes (biophysics) and on light scattering from large particles. He obtained his Ph.D. on liquid metals and alloys in 1983 also at the Free University of Berlin. Thereafter he joined the group of Professor P. Bennema in Nijmegen, The Netherlands, where he worked on growth of organic crystals from solution and melt. Since 1987 he has been in charge of the micromechanics group at the University of Twente. His research interest is now focused on resonator sensors, micro liquid handling systems such as pumps, valves, channels, mixers and microliquid flow sensors, basic physical-chemical mechanisms in key technologies in wet chemical etching and new actuator principles for applications in microrobotics. 